\title{
Brainstem Tuberculoma Presenting As Stroke
}

\author{
Vinod K.S. Gautam ${ }^{1}$, Ravinder Singh ${ }^{2}$, Sarbjeet Khurana ${ }^{3}$ \\ ${ }^{1}$ Department of Neurosurgery, ${ }^{2}$ Medical Anthropology, ${ }^{3}$ Epidemiology, \\ Institute of Human Behaviour \& Allied Sciences, Dilshad garden, Delhi, India
}

\begin{abstract}
An eighty year old male presented in the hospital with history of sudden onset weakness of the limbs and slurring of speech, features suggestive of stroke. The detailed clinical work up and radiological investigations revealed the presence of brainstem tuberculoma with hydrocephalus. He was treated with urgent ventriculoperitoneal shunt surgery and antitubercular chemotherapy. Patient had clinical and radiological improvement following surgery. After a period of one year of anti tubercular therapy and follow up, patient recovered completely and CT scan brain did not reveal any evidence of tuberculoma. The goal of this article is to enlighten readers about the possible presentations of Brainstem tuberculosis along with review of literature.

Keywords - Brainstem tuberculoma, CNS TB, Stroke
\end{abstract}

\section{INTRODUCTION}

The brainstem tuberculomas are least common in all the intracranial tuberculomas[1] . About 10\% of pulmonary Koch's patients develop central nervous system tuberculosis (CNS TB). It can present as lesions in brain or spine [2-4]. Tuberculoma, an uncommon manifestation of CNS TB usually causes seizures and focal signs. The clinical and radiological manifestations of tuberculoma are variable and can cause difficulty in the diagnosis in the absence of systemic tuberculosis or tubercular meningitis [2]. Early diagnosis is valuable for decreasing morbidity and preventing mortality.

\section{A CASE REPORT}

Eighty year old male, a resident of New Delhi presented with slurring of voice, difficulty in swallowing and weakness of all 4 limbs of sudden onset. He was a known case of hypertension on irregular medication. Patient was advised contrast enhanced Computed Tomography (CECT) of brain by a physician who revealed a hyper dense lesion in brainstem and was referred to our hospital as a case of hemorrhagic infarct. General examination revealed right sided nasolabial flattening suggestive of supra nuclear seventh cranial nerve paresis and impaired cough reflex. Motor examination revealed hypertonia and hyper reflexia in all limbs and $4 / 5$ power in both upper and lower limbs. Patient's lab investigations revealed raised erythrocyte sedimentation rate (E.S.R.) 60 at first hour. Magnetic Resonance Imaging of brain with Gadolinium enhancement ( Fig.1 and 2) revealed multiple ring enhancing thick walled lesions with disproportionate edema and central hypodensity in bilateral parietal regions, right temporo-occipital region and in the Pons of the brainstem with dilated lateral ventricles. Magnetic resonance spectroscopy (MRS) was strongly suggestive of tubercular etiology. These findings were suggestive of tubercular granuloma in view of the endemic nature of tuberculosis in India. There was no evidence of primary lung infection. Patient was put on antitubercular chemotherapy along with antiepileptic medication, acetazolamide and dexamethasone. He underwent right sided medium pressure ventriculo-peritoneal shunt for hydrocephalus. Postoperative period was uneventful and patient was discharged in stable condition. Early post operative follow-up was satisfactory and patient showed improvement in the power of both upper and lower extremities and facial paresis. After completion of four drug anti-tubercular therapy with Isoniazid, Rifampicin, Pyrizinamide and Ethambutol for two months and Rifampicin and Isoniazid for 10 months, patient had no neurological deficit and follow up CT scan (Figure-3) showed ventricular end of the shunt in situ with normal size of the ventricles and complete resolution of the tuberculomas.

\section{Discussion}

In earlier studies, the incidence of CNS tuberculoma in Britain has been found to be about $0.15 \%$ of space occupying lesions in brain [5-6] compared to $20 \%$ to $30 \%$ in India [7] .Tuberculosis accounts for about $5 \%$ of extra pulmonary cases in United States. Presentation of brainstem tuberculoma can be variable. Different types of presentations have been reported in literature including one and a half syndrome, mimicking glaucoma or glioma. It has also been reported to present as unilateral paralysis of saccades, Millard Gubler syndrome, isolated bilateral ptosis, horizontal gaze palsy, myokemia and facial contracture or as Foville's syndrome. A similar presentation, as seen in our case has been reported where medulla oblongata tuberculoma presented with stroke like symptoms and mimicked metastasis [8]. 


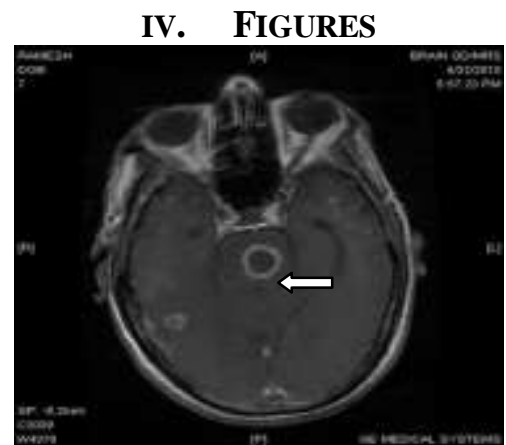

Figure -1. Pre operative T1W1 Gadolinium enhanced axial view of MRI of the brain showing a ring shaped lesion with central hypointensity located in the Pons of the brainstem

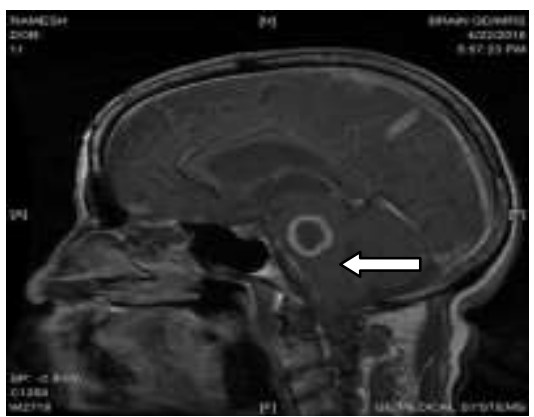

Figure- 2. Pre operative T1W1 Gadolinium enhanced sagittal view of MRI of the brain showing a ring shaped lesion with central hypointensity located in the Pons of the brainstem

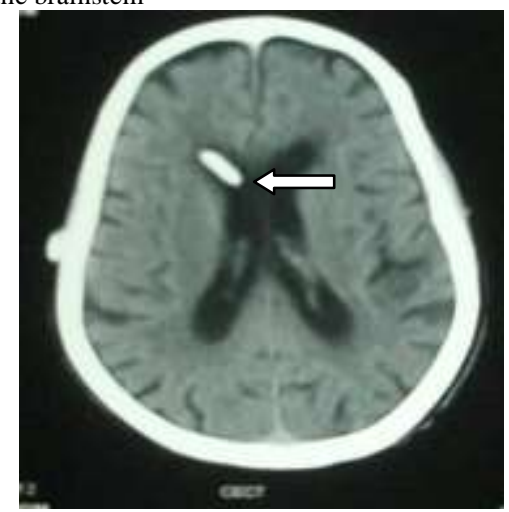

Figure-3. Post-operative follow up CT scan of the brain showing tip of the ventriculoperitoneal shunt in the frontal horn of the lateral ventricle and absence hydrocephalus and tuberculoma

\section{CONCLUSION}

In our country tuberculosis is endemic therefore brain-stem tuberculoma should be suspected in patients with space-occupying lesions of the brain stem. MRI of the brain with Gadolinium enhancement and MR spectroscopy helps in establishing the diagnosis and distinguishing tuberculomas from the verebrovascular accident (Stroke or Brain Attack). Early diagnosis and prompt therapy are important in preventing mortality and reducing morbidity.

\section{REFERENCES}

[1] Arseni, C. Two hundred and one cases of intracranial tuberculosis treated surgically. Journal of Neurology Neurosurgical Psychiatry, 1958; 21:308-11.

[2] Wasay M., Kheleani BA, Moolani MK, et al. Brain CT and MRI findings in 100 consecutive patients with intracranial tuberculoma. Neuroimaging. 2003; 13(3):240-47.

[3] Wang KC, Lin SM, Chen, Y., Tseng, S.H. Multiple tuberculous brain abscesses. Scandinavian Journal of Infectious Diseases, 2002; 34(12):931-34.

[4] Skendros, P., Kamaria, F., Kontopoulos, V. et al. Intradural, extramedullary tuberculoma of the spinal cord as a complication of tuberculous meningitis. Infection, 2003; 31(2):115-17.

[5] Maurice-Williams RS: TIberculomas of the brain in Britain. Postgraduate Medical Journal, 1972; 48:678-81.

[6] Dastur, H.M., Desai AD. A comparative study of brain tuberculomas and gliomas based on 107case record of each. Brain, 1965; $88: 375$.

[7] Ramaruthi, B., Varadajan MG. Diagnosis of tuberculomas of the brain. Journal of Neurosurgery, 1961; 18:1-7.

[8] Donmez FY, Coskun M, Guven G. Medulla oblongata tuberculoma mimicking metastasis presenting with stroke-like symptoms. Neurological Sciences, 2009; 30(4):349-52 